\title{
CAMBIOS RECIENTES DE USOS DEL SUELO Y SU POSIBLE RELACIÓN CON LAS TASAS DE CALENTAMIENTO EN LA ESPAÑA PENINSULAR
}

\author{
Celia SALINAS-SOLE ${ }^{1,2}$, Dhais PEÑA-ANGULO ${ }^{1,2}$, Ángel PUEYO-CAMPOS ${ }^{1,2}$, \\ Carlos LOPEZ-ESCOLANO ${ }^{1,2}$, José Carlos GONZALEZ-HIDALGO ${ }^{1,2}$ \\ ${ }^{1}$ Departamento de Geografía, Universidad de Zaragoza, España. \\ ${ }^{2} I U C A$, Universidad de Zaragoza, España.

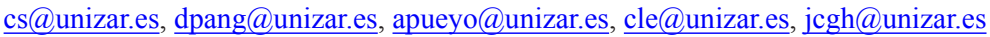

\section{RESUMEN}

Durante la segunda mitad del siglo XX en la España peninsular se ha registrado una tendencia positiva generalizada de las temperaturas, variable en su intensidad, distribución espacial, medida termométrica (máxima o mínima) y estación del año.

Paralelamente, durante las décadas recientes en España se ha asistido a profundas transformaciones de los paisajes con la modificación de extensas áreas por urbanización, infraestructuras, regadíos, incendios etc.

En general se acepta que las temperaturas diurnas (Tmax) dependen de factores globales, mientras las nocturnas (Tmin) dependen en mayor medida de factores locales, especialmente cambios de usos del suelo.

En el presente trabajo se analiza la posible relación entre las tasas de calentamiento observadas y los cambios de uso del suelo observados en la España peninsular evaluados por diferentes grados de accesibilidad, como indicador de dichos cambios. El estudio utiliza la base de datos de temperaturas promedio mensuales de Tmax y Tmin, MOTEDAS (resolución 10x10 km) y se enmarca dentro del proyecto DESEMON.

Los primeros resultados (periodo 1960-2010) sugieren una menor relación entre las superficies artificiales evaluadas por la accesibilidad y Tmax, mientras que se detecta una relación positiva con Tmin en las cuatro estaciones y el promedio anual, lo que pudiera sugerir algún tipo de efecto de la transformación de los paisajes sobre las temperaturas nocturnas.

Palabras clave: temperatura máxima, temperatura mínima, accesibilidad, España.

\begin{abstract}
During the second half of the 20th century on mainland Spain, there is a positive trend widespread of the temperatures, variable in the intensity, special distribution, maximum and minimum and the seasonality.

Generally, the change in the maximum temperatures (Tmax) is related to global factors meanwhile the night temperatures (Tmin) are associated with local factors, especially with the land use changes.

In this research, we analyze the relationship between the warming rates observed and the land use change assessed through the different levels of the accessibility index.
\end{abstract}


In this case, we are using the MOTEDAS dataset (resolution $10 \times 10 \mathrm{~km}^{2}$ ), for monthly average maximum and minimum temperatures created within the DESEMON project.

The first results for the period 1960-2010 suggest that there is a lower relationship between the artificial surfaces evaluated in the accessibility index and the Tmax, while is found a positive relationship with the Tmin in all of the seasons and with the annual average, this may suggest some kind of effect of the accessibility in the night temperatures.

Key words: maximum temperature, minimum temperature, accessibility, Spain.

\section{INTRODUCCIÓN}

En las décadas más recientes diversos análisis de las temperaturas han mostrado novedades de interés respecto al incremento térmico señalado con anterioridad. Así, se han constatado tendencias negativas de las temperaturas en extensas áreas, especialmente en EEUU y China; en otras ocasiones la tasas de evolución de las mínimas ha sido superior a la de las máximas, y por último en las décadas recientes se ha sugerido la existencia de un hiato térmico, ante la ausencia de significación de sus tendencias (Meehl, 2015).

Los argumentos sugeridos para intentar explicar estos tres hechos son diversos. Mientras el hiato se trata de explicar atendiendo a factores globales (GonzálezHidalgo et al., 2015a), en el caso de los otros dos se alude a factores generales como la calidad de los datos, su densidad y los efectos de determinados factores locales en las medidas termométricas (Capparelli et al., 2013). Dentro de esta discusión, cobra especial relevancia la evolución de la amplitud diaria que refleja la relación entre las tasas de las temperaturas máximas (Tmax) y mínimas (Tmin). La Tmax y Tmin reflejan las mediciones de temperatura diurna y nocturna y en consecuencia los efectos de los factores que controlan ambas mediciones (Mahmood et al., 2010). En general se acepta que la temperatura diurna de la superficie, es decir, Tmax, se debe principalmente a factores globales destacando la radiación solar sobre la superficie modulada por la cobertura de nubes, y la cantidad de calor liberado por el calor latente de evaporación de la humedad del suelo (Dai et al., 1999; Lauritsen y Rogers, 2012). Pero no está tan claro el comportamiento de la temperatura nocturna (es decir Tmin) debido a que las temperaturas nocturnas de la superficie se refieren a una capa de la atmósfera muy delgada cerca del suelo donde los flujos de calor latente pueden ser modificados por el efecto de los factores más locales (Kloztbach et al., 2009).

En el territorio peninsular español en las décadas recientes se han realizado numerosas investigaciones sobre la evolución de las temperaturas, que coinciden en señalar que desde mediados del siglo pasado se ha producido un aumento global especialmente en primavera y verano tanto en Tmax y Tmin (del Río et al., 2011; Ríos et al., 2012; Guijarro, 2013; González-Hidalgo et al., 2015b). Este aumento está en consonancia con otras áreas del Mediterráneo como Italia (Brunetti et al., 2006) y Grecia (Feidas et al., 2004). Sin embargo recientes estudios realizados con alta resolución espacial han sugerido que el aumento de las temperaturas no ha sido homogéneo en el tiempo, con máximo identificado en las décadas 1970-1990; se han detectado diferencias espaciales con los máximos valores localizados en la mitad sur y costa mediterránea, y por último tampoco en el tiempo ha habido uniformidad, pues se han observado 
claras diferencias temporales en las tendencias mensuales y estacionales tanto en la evolución de las temperaturas diurnas como nocturnas.

Estos resultados plantean problemas novedosos y no investigados en detalle hasta el momento. Por ejemplo, la evolución reciente y las variaciones espaciales detectadas en primavera y especialmente en verano de Tmax se han asociado a los bajos valores de humedad por bloqueos atlánticos en las décadas más recientes que habrían amortiguado el efecto del vapor de agua en la absorción de la radiación de onda larga terrestre (Philipona et al., 2005; Vicente-Serrano 2015; González-Hidalgo et al. 2016). Sin embargo, estos argumentos no son capaces de explicar el comportamiento de Tmin durante las últimas décadas.

Durante las décadas más recientes en el territorio peninsular español se han producido cambios drásticos en el uso del suelo relacionados con el regadío (Grindlay et al., 2011), las infraestructuras y la urbanización (Zúñiga et al., 2012), y los incendios forestales (Enríquez y del Moral, 2012), entre otros. Un segundo cambio ocurrido en los paisajes españoles durante las décadas recientes ha sido la introducción de superficies artificiales, de las que se reconoce su efecto diferenciado en las máximas y mínimas (Karl et al., 1988; Kalnay y Cai, 2003).

En el presente trabajo se presentan los primeros resultados del análisis de las relaciones entre cambios de usos del suelo recientes y evolución de las temperaturas máximas y mínimas en el territorio peninsular español. El estudio trata de indagar si existe relación entre la tasa de evolución térmica de las máximas y mínimas con indicadores de cambios de usos del suelo. En este estudio se analizará la relación entre un indicador que representa la transformación de las superficies "naturales" en superficies artificiales, el índice de accesibilidad, y la tasa de tendencia de las máximas y mínimas estacionales. El trabajo es el inicio de una investigación enfocada a conocer las relaciones entre diversos indicadores de cambios de paisaje y la evolución de las temperaturas en las décadas recientes.

\section{MÉTODOS}

En el presente estudio se emplearán las series estacionales de los promedios mensuales de Tmax y Tmin procedentes de la malla de alta resolución de la base de datos de temperaturas medias mensuales de España (MOnthly TEmperature DAtaset of Spain) denominada MOTEDAS (Gonzales-Hidalgo et al., 2015a). Esta malla procede del vaciado y depuración de los fondos documentales de AEMet y se ha calculado con 1300 observatorios libres de anómalos, homogeneizados con series de referencias y rellenados, siendo seleccionados los incluidos en la malla por el porcentaje de datos originales y la cercanía de los datos faltantes reconstruidos.

Los cambios en el uso del suelo se ha analizado mediante el índice de accesibilidad que combina el potencial de población, el desarrollo de una red viaria sobre todo de alta capacidad y el desarrollo urbano, entre 1960-2010 (López-Escolano et al. 2015). La accesibilidad así definida es un indicador de la facilidad con la que se puede acceder a actividades desde una localización determinada a través de un sistema de transporte (Morris et al., 1979), y se convierte en un indicador de las transformaciones superficiales de los paisajes. En su cálculo se han considerado la totalidad de los municipios de territorio peninsular español y la red viaria que conecta la totalidad de las cabeceras 
municipales durante el periodo seleccionado. Así, además de las redes principales del Plan General de Carreteras 1984-1991, y del Plan Estratégico de Infraestructuras de Transporte 2005-2020 que se ciñeron exclusivamente a la parte estructurante de la Red de Interés General del Estado (MOPTMA, 1994; PEIT, 2005), se consideraron otras vías de gran capacidad como autopistas y autovías. Debido a la pluralidad de estas bases se categorizó la red en cuatro niveles para favorecer el análisis.

El índice de accesibilidad original está calculado en una malla de $5 \times 5 \mathrm{~km}$ y en diferentes años $(1960,1968,1983,1995)$ y difiere de la resolución de la malla de temperaturas $(10 \times 10 \mathrm{~km})$. Por esta razón, y con el fin de homogeneizar espacial y temporalmente las mallas de ambas variables, la malla de accesibilidad se convirtió a la resolución de MOTEDAS por medio de la media de los cuatro pixel correspondientes. La homogeneidad temporal se resolvió recalculando en MOTEDAS las tasas de tendencias de las temperaturas estacionales del periodo común 1960-2010, y estimando la tasa de cambio de accesibilidad entre ambas fechas como estimador de su tendencia.

El signo (positivo/negativo) y la significación $(\mathrm{p}<0,05)$ de las tendencias de Tmax y Tmin se han evaluado mediante el test de Mann-Kendall (Mann, 1945) y la magnitud o tasa con el estadístico de Sen (Sen, 1968). Para asegurar la independencia de cada serie mensual de temperaturas se analizó la posible existencia de autocorrelación, y en aquellas celdas y series en las que se detectó fue filtrada con el método denominado trend-free pre-whitening (TFPW) propuesto por Yue et al. (2002).

La relación entre los cambios de la accesibilidad y de la temperatura se ha analizado mediante el coeficiente de Pearson, considerando como variables la variación de la accesibilidad y la tasa de Sen en el periodo estudiado 1960-2010. Aunque el análisis se realizó en toda la malla, los resultados se han filtrado correlacionando solamente las celdas que tenían información original de temperatura (1130 celdas).

\section{RESULTADOS}

Durante el periodo 1960-2010 la tasa de variación de la accesibilidad es más elevada en toda la zona de litoral, tanto en la vertiente mediterránea como en el extremo noroeste atlántico, en Galicia, así como en los corredores que conectan Andalucía y Valencia con el centro de la península o el del valle del Ebro (Figura 1). En general las zonas de variación más baja se corresponden con las áreas de montaña (Cantábrica, Pirineos, Ibérica, Béticas) y extensas áreas de interior especialmente la meseta norte y áreas de la meseta sur.

En el mismo periodo las tendencias de las temperaturas son mayoritariamente positivas si bien sus tasas varían entre los distintos momentos del año. En primavera y verano las tasas de Tmax y Tmin son positivas y significativas en la mayor parte del territorio peninsular (un 95\% de la superficie, $\mathrm{p}<0$ ’05 en el caso de las mínimas). En el invierno un $60 \%$ de la superficie tiene tendencias significativas en sus temperaturas máximas. La distribución de las áreas con tendencias no significativas difiere en Tmax: durante el invierno se localizan preferentemente en la mitad este; en primavera en la mitad sur y en verano en el cuadrante noroeste (Figura 2). En Tmin cabe destacar que las áreas con tendencias significativas en otoño se localizan en la mitad sur y costa mediterránea (Figura 3). Estos resultados referidos al periodo 1960-2010 suponen variaciones respecto a las tendencias descritas en el periodo completo de MOTEDAS 1951-2010 en invierno y primavera. 


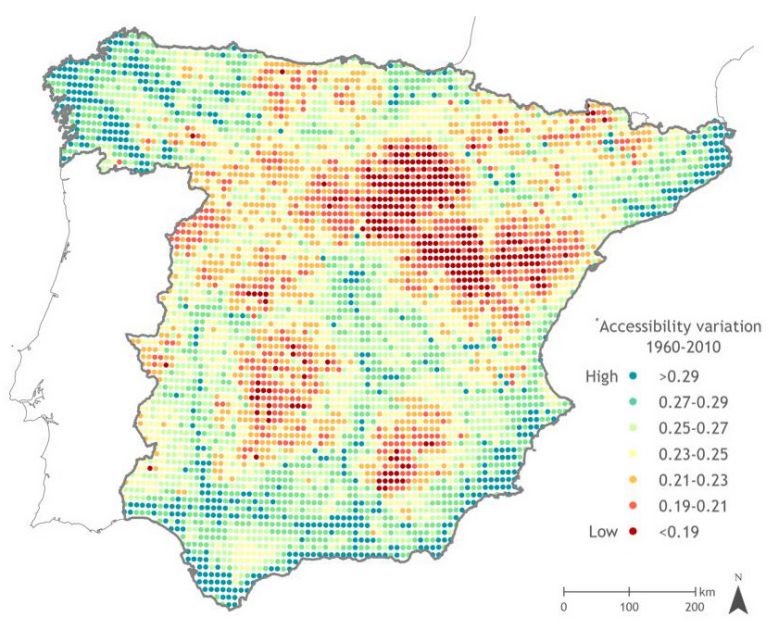

Figura. 1: Variación del índice de accesibilidad (1960-2010). Fuente: elaboración propia.

La distribución espacial de la intensidad del calentamiento de las temperaturas mínimas es homogénea en primavera y en verano en todo el territorio, mientras que los valores más altos en el invierno se localizan en el sur y en el noreste de la península. Los valores promedio estacionales de las máximas oscilan entre $0,34^{\circ} \mathrm{C} /$ década en primavera y $0,06^{\circ} \mathrm{C} /$ década en otoño, mientras en las mínimas varían entre $0,36^{\circ} \mathrm{C} /$ década en verano y $0,14^{\circ} \mathrm{C} /$ década en invierno. Estos valores no obstante deben tomarse con cierta cautela dado que excepto en primavera y verano la superficie afectada por tendencias no significativas puede ser muy extensa.

La relación entre las tasas de calentamiento y el índice de accesibilidad se muestra en la Figura 2. Los resultados indican que existen diferencias en la relación entre la tasa de calentamiento y las variaciones de la accesibilidad de las temperaturas diurnas y nocturnas. En las máximas la relación tiene signo negativo excepto en otoño, y la máxima correlación se ha encontrado en primavera. En las mínimas, la tasa de variación se correlaciona positivamente con las tasas de variación de la accesibilidad en las cuatro estaciones, con valores superiores (salvo primavera) a los de las máximas (Tabla 1).

\begin{tabular}{|c|c|r|r|r|r|}
\cline { 3 - 6 } \multicolumn{1}{c|}{} & \multicolumn{1}{c|}{ Invierno } & Primavera & \multicolumn{1}{c|}{ Verano } & \multicolumn{1}{c|}{ Otoño } \\
\hline \multirow{3}{*}{ Máximas } & $\mathrm{b}$ & -0.3679 & -0.701 & -0.5074 & 0.0497 \\
\cline { 2 - 6 } & $r$ & $-0,1863$ & $-0,2874$ & $-0,19$ & 0,0224 \\
\cline { 2 - 6 } & $p$ & $2.555 \mathrm{e}-10$ & $2.2 \mathrm{e}-16$ & $1.224 \mathrm{e}-10$ & 0.454 \\
\hline \multirow{3}{*}{ Mínimas } & $\mathrm{b}$ & 0.3222 & 0.4721 & 0.8784 & 0.767 \\
\cline { 2 - 6 } & $r$ & 0,2131 & 0,2412 & 0,314 & 0,3641 \\
\cline { 2 - 6 } & $p$ & $4.439 \mathrm{e}-13$ & $2.2 \mathrm{e}-16$ & $2.2 \mathrm{e}-16$ & $2.2 \mathrm{e}-16$ \\
\hline
\end{tabular}

Tabla. 1: Coeficiente de correlación de Pearson (r) Pendiente del modelo de regresión lineal simple (b) P-value ( $p$ ) entre la variación de la accesibilidad y la tasa de Tmax y Tmin (1960-2010). Fuente: elaboración propia. 

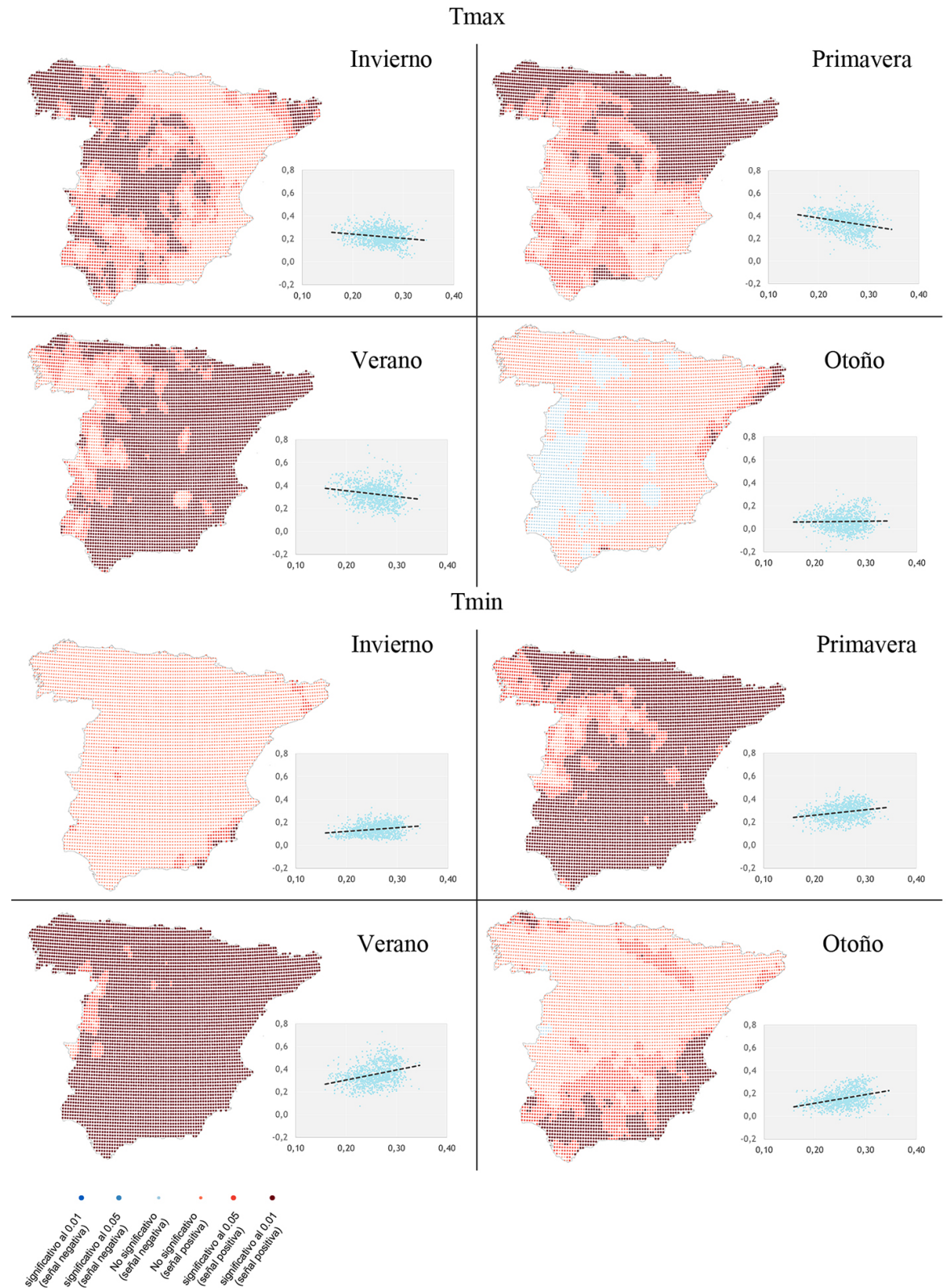

Figura. 2: Significación de las tendencias estacionales y relación entre variaciones de la accesibilidad y tasa de variación de Tmax y Tmin (1960-2010). Fuente: elaboración propia.

Un segundo aspecto se refiere a la intensidad de esta relación, evaluada mediante el modelo de regresión lineal, cuyos coeficientes (Tabla 1) indican que la fortaleza de dicha relación especialmente en las mínimas es más intensa en verano y otoño. 


\section{DISCUSIÓN Y CONCLUSONES}

El análisis de la evolución de las temperaturas promedio mensuales mínimas en el periodo 1960-2010 indica que su tasa global ha sido superior a la de las máximas y por ello en amplias zonas del territorio la amplitud térmica sigue siendo negativa. Esta tendencia de la amplitud ha sido generalizada hasta la década de los ochenta del pasado siglo (Karl et al., 1993), pero en fechas recientes se ha sugerido un cambio de su tendencia general a signo positivo (Fall et al., 2011; Makowsky et al., 2008), que sin embargo no concuerda con la tendencia negativa detectada en numerosas zonas del planeta, como en el este de África (Christy et al., 2009), EE.UU. (Fall et al., 2011; Cordero et al., 2011; Tang et al., 2013; Misra y Michael, 2013), Canadá (Vincent et al., 2012), Moldavia (Corobov et al., 2010), China (Wu y Yang, 2013), o Península Ibérica (González-Hidalgo et al., 2015a) entre otros lugares. El hecho de que se mantenga una tendencia negativa de la amplitud implica que la tasa de variación de la temperatura mínima es superior a la diurna, y devuelve una vez más el debate a los factores que controlan ambas medidas termométricas.

En los últimos treinta años los paisajes del territorio peninsular español han sido sometidos a cambios intensos y extensos. Las zonas de nuevo regadío por ejemplo, que representan el 7\% de la superficie total, aumentaron alrededor de un 50\% entre 1961-2011, cuyos efectos no solo sugieren modificaciones de los flujos de calor sensible y latente, sino también localmente el albedo. Pero también la introducción de superficies artificiales ha afectado a extensas áreas y no solo mediante la urbanización, factor muy debatido (véase Jones et al., 2008), sino en el caso analizado en el presente estudio por la transformación de la red de infraestructuras y la introducción de superficies artificiales en el paisaje. Karl et al. (1988) sugirieron que la urbanización en los EE.UU. disminuyó Tmax, excepto en invierno, al tiempo que aumentaba la tasa de Tmin en todas las estaciones del año, originando que la tendencia de la amplitud térmica fuese negativa en las cuatro estaciones. Del mismo modo, Kalnay y Cai (2003) relacionaron una parte significativa del aumento de la temperatura superficial en el sureste de los EE.UU. con los procesos vinculados al desarrollo, y sugirieron que el $50 \%$ del calentamiento total era debido a la urbanización.

La construcción de infraestructuras en las últimas tres décadas en la España peninsular ha sido espectacular, sobre todo en las zonas del este y la costa, en muchas ocasiones en paralelo a procesos de urbanización (no analizados en el presente estudio). Los resultados del presente análisis parecen sugerir que estas alteraciones han podido afectar la evolución de las temperaturas nocturnas pero no las diurnas.

Esta conclusión provisional sugeriría que los registros de mínimas pueden estar reflejando en una medida no evaluada por el momento el efecto de factores locales como se ha sugerido en anteriores estudios, ligados en el caso del presente a superficies artificiales, capaces de alterar los flujos de calor sensible principalmente (cabe pensar que el calor latente movilizado desde estas superficies sea negligible por su naturaleza).

Las extensas transformaciones de los paisajes en el territorio español peninsular durante las décadas más recientes sugieren que aquellas pueden tener un efecto diferenciado sobre la evolución de las temperaturas máximas y mínimas que requiere ser investigados con mayor profundidad y detalle. La investigación en curso se encamina 
a evaluar otros indicadores de transformación de los paisajes con el objeto de poder valorar con mayor detalle dichos efectos.

\section{AGRADECIMIENTOS}

Ministerio de Ciencia e Innovación, Gobierno de España, Proyecto DESEMON, CGL2014-52135-C3-3-R. Gobierno Regional de Aragón DGA-FSE (Grupo de Investigación Consolidado "Clima, Agua, Cambio Global y Sistemas Naturales"). Dhais Peña-Angulo y Celia Salinas-Solé son becarias de doctorado del Programa FPI del Ministerio de Ciencia e Innovación. Datos climáticos originales cedidos por AEMet.

\section{REFERENCIAS}

Brunetti, M.; Maugeri, M.; Monti, F.; Nanni, T. (2006). Temperature and precipitation variability in Italy during the last two centuries from homogenized instrumental time series. International Journal of Climatology, 26, 345-381. doi: http://dx.doi. org/10.1002/joc. 1251

Capparelli, V.; Franzke, C.; Vecchio, A.; Freeman, M.P.; Watkins, N.W.; Carbone, V. (2013). A spatio-temporal analysis of US station temperature trends over the last century. Journal of Geophysical Research-Atmospheres, 118, 7427-7434. doi: http://dx.doi.org/10.1002/jgrd.50551

Corobov, R.; Sheridan, S.; Overcenco, A.; Terinte, N. (2010). Air temperature trends and extremes in Chisinau (Moldova) as evidence of climate change. Climate Research, 42, 247-256. doi: http://dx.doi.org/10.3354/cr00922.

Dai, A.; Trenberth, K.E.; Karl, T. (1999). Effects of clouds, soil moisture, precipitation, and water vapor on diurnal temperature range. Journal of Climate, 12, 2451-2473.

del Rio, S.; Herrero, L.; Pinto-Gomes, C.; Penas, A. (2011). Spatial analyses of mean temperature trends in Spain over the period 1961-2006. Global Planetary Change, $78,65-75$.

Enríquez, E.; Del Moral, L. (2012). Los Incendios Forestales en España. Decenio 2001-2010. Ministerio de Agricultura, Alimentación y Medio Ambiente. Madrid

Fall, S.; Watts, A.; Nielsen-Gammon, J; Jones, E; Niyogi, D; Christy, JR; Pielke Sr RA. (2011).Analysis of the impacts of station exposure on the US Historical Climatology Network temperatures and temperature trends. Journal of Geophysical ResearchAtmospheres 116, D14120. doi: http://dx.doi.org/10.1029/2010JDO15146.

Feidas, H; Makrogiannis, T; Bora-Senta, E. (2004). Trend analysis of air temperature time series in Greece and their relationship with circulation using surface and satellite data: 1955-2001. Theoretical and Applied Climatology, 79, 185-208.

González-Hidalgo, J.C; Brunetti, M; de Luis, M. (2011). A new tool for monthly precipitation analysis in Spain: MOPREDAS database (Monthly precipitation trends December1945-November 2005). International Journal of Climatology, $31,715-731$.

González-Hidalgo, J.C.; Peña-Angulo, D.; Brunetti, M.; Cortesi, N. (2015a). MOTEDAS: A new monthly temperature database for mainland Spain and the trend in temperature (1951-2010). International Journal of Climatology, 31, 715 731. doi: http://dx.doi.org/10.1002/joc.4298 
González-Hidalgo, J.C.; Peña-Angulo, D.; Brunetti, M.; Cortesi, N. (2015b). Recent trend in temperature evolution in Spanish mainland (1951-2010): from warming to hiatus. International Journal of Climatology doi: http://dx.doi.org/10.1002/ joc. 4519 .

Grindlay, AL; Lizárraga, C; Rodríguez, M.I.; Molero, E. (2011). Irrigation and territory in the southeast of Spain: evolution and future perspectives within new hydrological planning. WIT Transactions on Ecology and the Environment, 150, 623-638.

Guijarro J.A. (2013). Tendencias de la Temperatura. In Garcia-Legaz C, Valero F (eds). Fenómenos meteorológicos adversos en España, WCRP, AMV Ediciones: Madrid, 313-323.

Jones, PD; Lister, DH; Li, Q. (2008). Urbanization effects in large-scale temperature records with an emphasis on China. Journal of Geophysical research, 113, D16122.

Kalnay, E; Cai, M. (2003). Impact of urbanization and land-use change on climate. Nature, 423, 528-531.

Karl, T.R; Diaz, H.F; Kukla, G. (1988). Urbanization: its detection and effects in the United Stated climate records. Journal of Climate, 1, 1099-1123.

Karl, T.R; Jones, P.D; Knight, R.W.; Kukla, G; Plummer, N; Razuvayev, V; Gallo, K.P; Lindseay, J; Charlson, RJ; Peterson, T.C. (1993). Asymmetric trends of daily maximum and minimum temperature. Bulletin American Meteorological Society, 74, 1007-1023.

Klotzbach, P.J; Pielke, R.A. Sr; Pielke, R.A. Jr; Christy, J.R; McNider, R.T. (2009). An alternative explanation for differential temperature trends at the surface and the lower troposphere. Journal of Geophysical Research, 114, D21102, doi: http:// dx.doi.org/10.1029/2009JD011841.

Lauritsen, R.G; Rogers, J.C. (2012). U.S. Diurnal Temperature Range Variability and Regional Causal Mechanisms, 1901-2002. Journal of Climate, 25, 7216-7231.

López-Escolano, C; Pueyo-Campos, A; Postigo-Vidal, R; Alonso-Logroño, M.P. (2015). Representación cartográfica de redes viarias e indicadores de accesibilidad para series cronológicas amplias: el caso de la España peninsular 1960-2014. In: de la Riva, J; Ibarra, P; Montorio, R; Rodrigues, M. (Coord.), Análisis espacial y representación geográfica: innovación y aplicación (pp. 205-214). Zaragoza: XXIV Congreso de la Asociación Española de Geógrafos.

Mahmood, R ; Pielke, R.A. Sr ; Hubbard, K.G. (2010). Impact of land use/land cover change on climate and future research priorities. Bulletin American Meteorological Society pp.37-46.

Makowsky, K; Wild, M; Ohmura, A. (2008). Diurnal temperature range over Europe between 1950-2005. Atmospheric Chemistry and Physics, 8, 6483-6498.

Mann, H.B. (1945). Non parametric test against trend. Econometrica, 13 (3), 245-259. doi: http://dx.doi.org/10.2307/1907187

Meehl, G.A. (2015). Decadal climate variability and the early-2000s hiatus. US Clivar Variations, 13, 1-6. 
Philipona, R; Dürr, B; Ohmura, A; Ruckstuhl, C. (2005). Anthropogenic greenhouse forcing and strong water vapor feedback increase temperature in Europe. Geophysical Research Letter, 32, L19809, doi: 10.1029/2005GL023624, 2005

Rios, D; Penas, D; del Rio, S. (2012). Comparative analysis of mean temperature trends in continental Spain over the period 1961-2010. International Journal of Geobotanical Research, 2, 41-55.

Sen, P.K. (1968). Estimates of the regression coefficient based on Kendall's tau. Journal of the American Statistical Association, 63 (324), 1379-1389. doi: http:// dx.doi.org/10.2307/2285891

Vincent, L.A; Wang, X.L; Milewska, E. (2012). A second generation of homogenized Canadian monthly surface air temperature for climate trend analysis. Journal of Geophysical Research Letter-Atmospheres, 117, D18110. doi: 10.1029/2012JD017859.

Wu, K; Yang, X. (2013). Urbanization and heterogeneous surface warming in eastern China. Chinese Science Bulletin. doi: 10.1007/s

Yue, S; Pilon, P; Phinney, B; Cavadias, G. (2002). The influence of autocorrelation on the ability to detect trend in hydrological series. Hydrological Processes, 16 (9), 1807-1829. doi: http://dx.doi.org/10.1002/hyp.1095

Zúñiga, M; Pueyo, A; Calvo, J.L. (2012) The Spanish population during the 20th century and beyond. Journal of Maps, 8, 1-6 doi:10.1080/17445647.2012.744364 